\title{
Viscous Diffusion of Vorticity Using the Diffusion Velocity Concept*
}

\author{
J.H. Strickland, S.N. Kempka and W.P. Wolfe \\ Engineering Sciences Center \\ Sandia National Laboratories \\ Albuquerque, NM 87185-0836, USA
}

\begin{abstract}
The primary purpose of this paper is to provide an evaluation of the diffusion velocity concept with regard to its ability to predict the diffusion of vorticity near moving walls as well as in unbounded two-dimensional space. We also briefly outline a "bi-zonal" wall model which utilizes the diffusion velocity concept and is compatible with vortex methods. A primary goal of this model is to minimize the number of vortices generated on the surface at each time step while achieving good resolution of the vorticity field near the wall. We have generated results for an initially square patch of vorticity in an unbounded domain, an impulsively started flat plate, an oscillating flat plate, uniform flow over a semi-infinite flat plate, and flow over a wedge. The simulations are found to give excellent results when compared to classical solutions of these problems.
\end{abstract}

\section{Introduction}

\subsection{Background}

Our ultimate reason for doing this work is to be able to develop a robust algorithm which is compatible with vortex methods while at the same time yielding good resolution of the vorticity/ velocity field near the wall. Several attempts have been made in the past to couple inviscid vortex models with classical integral boundary layer formulations. Typical of these is the work due to Spalart and Leonard [13] and Spalart [12]. The integral solutions were started from a known stagnation point and proceeded in the stream-wise direction until separation was indicated. Slightly upstream of the separation point, vortices were introduced to replace the vorticity in the boundary layer and were then allowed to convect freely in the flow. While the method gives good results for simple boundary layer flows, it is difficult (if not impossible) to apply to complicated unsteady flows in which there are multiple stagnation, separation, and reattachment points which are moving as a function of time.

There are several methods in which the viscous diffusion process (not necessarily in thin boundary layers) has been modeled using Lagrangian vortex elements. These methods include the Gaussian random walk method [2], the diffusing core method [9], the particle strength exchange (PSE) method [5], and the diffusion velocity method originally proposed by Ogami and Akamatsu [10] and improved by Kempka and Strickland [6]. The relative merits of these methods are discussed in $[6]$.

${ }^{*}$ This work performed at Sandia National Laboratories supported by the U. S. Dept. of Energy under contract No. DE-AC04-94AL85000. 
Chorin [3] applied the "random walk" method to boundary layers utilizing vortex sheets near the wall. His method predicts a zero pressure gradient laminar boundary layer quite well but does not successfully predict separating boundary layers. This is probably due to his use of boundary layer assumptions near the separation point. For instance, the equation he used to calculate the stream-wise velocity as a function of distance from the wall neglects the stream-wise gradient of the normal velocity. Near the separation point this gradient is the same order of magnitude as the vorticity and cannot be neglected.

Koumoutsakos, Leonard and Pepin [8] recently applied the PSE method to flow around both an oscillating and impulsively started circular cylinder. They obtained excellent results for the range of Reynolds numbers studied (40 to 10000). It appears that the number of vortices $N_{v}$ used in their work scales roughly as $\sqrt{R e}$. This scaling is consistent with the assumption that the thickness of the boundary layer $\delta$, non-dimensionalized by a length $L$, scales as $1 / \sqrt{R e}$ and that the overlapping vortex elements are circular. The primary disadvantage of the PSE method is that the region near the boundary must initially be flooded with vortex elements whose size, number, and extent must be judicially chosen. As these elements convect in the flow, additional adjustments must be made in order to maintain a sufficient number of elements near the boundaries which are sized to properly resolve the boundary layer.

It should be noted that a topological problem exists if one tries to use circular vortex blobs to simulate boundary layers at high Reynold's Numbers. Consider a two-dimensional unsteady flow field which contains boundaries which are impenetrable. In order to solve the flow field, we first discretize the boundary into a number of curved or flat panels of length $O(h)$. In order to accurately model the diffusion process using circular blobs, we must place a large number of overlapping circular vortices along the wall whose diameters will be some fraction of the boundary layer thickness. For high Reynolds number flows the boundary layer thickness may itself be some fraction of $h$. In such cases, the number of vortex blobs required becomes prohibitive. We note that Chorin's use of vortex sheets in reference [3] was motivated by this very problem which became apparent in his earlier work [2] in which he used circular vortex blobs with finite cores.

\subsection{General methodology}

We now outline a two-dimensional unsteady viscous "bi-zonal" wall model which is compatible with vortex methods. We first define two zones, a wall region for $y \leq h$ and an outer region for $y>h$ where $y$ is the normal distance away from the wall and $h$ is the local panel length. It is assumed that the boundary is properly paneled in order to resolve the wall boundary conditions. In the wall region, elements used to represent the vorticity field must be elongated in the stream-wise direction as compared with their thickness normal to the wall. In the outer region, the vortex elements are circular with diameters of $O(h)$. In the wall region, diffusion may be assumed to be unidimensional so long as the wall curvature is not too severe, while in the outer region diffusion will be considered as two-dimensional. Finally, in the wall region, a Lagrangian re-map scheme is used to obtain the evolution of the vorticity field while in the outer region a viscous vortex blob method is used. The vortex elements defined in each region are able to smoothly interact with the elements of the other region or transition from one region to the other. This method is intended to yield good resolution of the diffusion and convection processes for $y<h$ without generating any "free" vortex blobs which are smaller than $O(h)$.

The general solution scheme for the wall region is to first diffuse vorticity into the layer, allow diffusion of the existing vorticity to occur, convect the flow, re-map the vorticity distribution back onto lines normal to the wall which are spaced at intervals $h$, and repeat for the next time step. In most regions of high Reynolds number boundary layer flows, only the wall region will be required 
since the diffusing layer will not extend beyond $y=h$. We note that for all of the calculations presented herein, this is the case. For cases where sufficient vorticity moves across $y=h$, the vorticity is converted into "free" circular vortex blobs of support $O(h)$. Conversely, vortex blobs may penetrate the wall layer in which case the vorticity from such blobs is "captured" and placed in the wall layer. We are presently developing numerical algorithms to model the outer zone and its interaction with the inner zone.

Near the wall, the diffusion process is modeled by using one-dimensional overlapping linear vortex elements as indicated in Fig. 1. It is possible to use other one-dimensional vortex elements in this region such as Gaussian or "skewed" Gaussian elements (e.g. different core radii for positive and negative positions with respect to the element center). However, the linear elements provide a much simpler model and do not suffer from typical problems near walls of distributions which do not have compact support (e.g. part of the distribution extends across the wall). These elements diffuse according to the "diffusion" velocity $\boldsymbol{u}_{d}$ at their edges and center. In order to conserve circulation strength, the area of each triangle under the $\omega, y$ curve remains constant as a function of time. Details of the diffusion velocity concept will be given in the next section.

Our next task is to convect the flow in the wall layer. For convection purposes we use quadrilateral Lagrangian elements. Due to the rapid distortion of the elements in the wall layer (especially next to the wall), the vorticity distribution is re-mapped at each iteration back onto lines which are normal to the wall. The tangential convection velocity may be obtained from the following equation which is based upon the definition of vorticity in terms of velocity.

$$
u=u_{w}-\int_{w}^{y} \omega d y+\int_{w}^{y} \frac{\partial v}{\partial x} d y
$$

Here the subscript denotes values at the wall. The normal component of the convection velocity may be obtained from the continuity equation as:

$$
v=v_{w}-\int_{w}^{y} \frac{\partial u}{\partial x} d y
$$

In closing this section, we acknowledge that the above outline which we have given for the solution of two-dimensional wall layers using a vortex method is very sketchy. As stated earlier, the primary work presented in this report concerns the simulation of viscous diffusion in the wall layer using the diffusion velocity concept. Our purpose for including the above outline is to orient the reader to the role which the diffusion sub-model will play in a more general scheme.

\section{Method of solution}

\subsection{The diffusion velocity concept}

In order to illustrate the diffusion velocity concept, consider the two-dimensional viscous flow field depicted in Fig. 2. The diffusion velocity is specified such that the circulation within a given boundary remains constant if that boundary moves at the diffusion velocity plus the local fluid velocity. In order to formulate the diffusion velocity we first note that the circulation around a curve enclosing an area $A$ is

$$
\Gamma=\int_{A} \boldsymbol{\omega} \cdot \hat{n} d A
$$


Taking the time derivative of Eq. 3 and setting it equal to zero yields:

$$
\frac{d \Gamma}{d t}=\int_{A}\left[\frac{\partial}{\partial t} \boldsymbol{\omega}+(\tilde{u} \cdot \nabla) \boldsymbol{\omega}+\boldsymbol{\omega}(\nabla \cdot \tilde{u})-(\boldsymbol{\omega} \cdot \nabla) \tilde{u}\right] \cdot \hat{n} d A=0
$$

where $\tilde{u}$ is the velocity at which the area $A$ occupied by vorticity is moving. We now let $\tilde{u}=\boldsymbol{u}+\boldsymbol{u}_{d}$ where the local fluid velocity is $\boldsymbol{u}$ and the local diffusion velocity is $\boldsymbol{u}_{d}$. Equation 4 is satisfied when the integrand is equal to zero:

$$
\frac{\partial}{\partial t} \boldsymbol{\omega}+(\tilde{u} \cdot \nabla) \boldsymbol{\omega}+\boldsymbol{\omega}(\nabla \cdot \tilde{u})-(\boldsymbol{\omega} \cdot \nabla) \tilde{u}=0
$$

We also have at our disposal the vorticity form of the Navier-Stokes equation:

$$
\frac{\partial}{\partial t} \boldsymbol{\omega}+(\boldsymbol{u} \cdot \nabla) \boldsymbol{\omega}+\boldsymbol{\omega}(\nabla \cdot \boldsymbol{u})-(\boldsymbol{\omega} \cdot \nabla) \boldsymbol{u}=\nu \nabla^{2} \boldsymbol{\omega}
$$

Subtracting Eq. 6 from Eq. 5 yields the governing equation for the diffusion velocity in terms of the vorticity field and kinematic viscosity:

$$
\left(\boldsymbol{u}_{d} \cdot \nabla\right) \boldsymbol{\omega}+\boldsymbol{\omega}\left(\nabla \cdot \boldsymbol{u}_{d}\right)-(\boldsymbol{\omega} \cdot \nabla) \boldsymbol{u}_{d}=-\nu \nabla^{2} \boldsymbol{\omega}
$$

By using the vector identities, $\nabla \times\left(\boldsymbol{u}_{d} \times \boldsymbol{\omega}\right)=(\boldsymbol{\omega} \cdot \nabla) \boldsymbol{u}_{d}-\left(\boldsymbol{u}_{d} \cdot \nabla\right) \boldsymbol{\omega}+\boldsymbol{u}_{d}(\nabla \cdot \boldsymbol{\omega})-\boldsymbol{\omega}\left(\nabla \cdot \boldsymbol{u}_{d}\right)$, $\nabla \cdot \boldsymbol{\omega}=0$, and $\nabla \times(\nabla \times \boldsymbol{\omega})=\nabla(\nabla \cdot \boldsymbol{\omega})-\nabla^{2} \boldsymbol{\omega}$, Eq. 7 can be written in a form in which the diffusion velocity appears only once:

$$
-\nabla \times\left(\boldsymbol{u}_{d} \times \boldsymbol{\omega}\right)=\nu \nabla \times(\nabla \times \boldsymbol{\omega})
$$

This equation implies that to within an arbitrary constant:

$$
\boldsymbol{u}_{d} \times \boldsymbol{\omega}=-\nu \nabla \times \boldsymbol{\omega}
$$

The arbitrary constant can be shown to be equal to zero. We now restrict ourselves to two dimensions for which Eq. 9 simplifies to:

$$
\boldsymbol{u}_{d}=-\frac{\nu}{\omega} \nabla \omega
$$

where $\omega$ is the vorticity which is perpendicular to the two-dimensional plane. In order to determine the time rate of change of the vorticity as we move along at the velocity $\tilde{u}$ (denoted by $\tilde{D} / \tilde{D} t$ ) we write Eq. 5 for the two-dimensional case:

$$
\frac{\tilde{D} \boldsymbol{\omega}}{\tilde{D} t}=-\boldsymbol{\omega}(\nabla \cdot \tilde{u})=-\boldsymbol{\omega}\left(\nabla \cdot \boldsymbol{u}_{d}\right)
$$

We see from this equation that the time rate of change of vorticity is a function of the divergence of the diffusion velocity since $\nabla \cdot \boldsymbol{u}=0$ for incompressible flow.

When we are dealing with thin boundary layers, the diffusion in the direction tangential to the wall (the $x$ direction) may be ignored. In this case, we only concern ourselves with the diffusion velocity in the direction normal to the wall (the $y$ direction) which will be denoted by $U_{d}$.

$$
U_{d}=-\frac{\nu}{\omega} \frac{\partial \omega}{\partial y}
$$

The evolution equation for $\omega$ may be obtained from Eq. 11 as:

$$
\frac{\tilde{D} \omega}{\tilde{D} t}=-\omega \frac{d U_{d}}{d y}
$$




\subsection{Generation of vorticity at the wall}

The circulation per unit length $\Delta \gamma$ which must be generated at the wall in order to maintain the no-slip condition is equal to the slip velocity $\Delta U_{s}$ which would otherwise become manifest at a particular location on the wall during a time period $\Delta t$. A generalized method to obtain $\Delta U_{s}$ (or $\Delta \gamma$ on the wall) is given in reference [7]. We can define the wall slip velocity $U_{s}$ at any instant in time as:

$$
U_{s} \equiv U_{w s}-U_{w f}
$$

where $U_{w s}$ is the tangential surface velocity or tangential velocity boundary condition and $U_{w f}$ is the tangential fluid velocity which are both positive in the direction of the unit surface tangent vector $\boldsymbol{\tau}_{s}$. In order to restore the no-slip condition at the wall during the increment of time $\Delta t$, a flux of vorticity must occur through the wall into the fluid. This flux can be written in terms of the diffusion velocity $U_{d}$ and the change in the wall slip velocity $\Delta U_{s}$ as:

$$
\omega U_{d} \Delta t=\Delta U_{s}
$$

or

$$
\omega U_{d}=\frac{d U_{s}}{d t}
$$

It should be noted that if the surface normal vector which is pointing into the flow of interest is $\boldsymbol{\eta}_{s}$, the positive direction associated with $\boldsymbol{\omega}$ in Eq. 16 is given by $\boldsymbol{\tau}_{s} \times \boldsymbol{\eta}_{s}$. Since the diffusion velocity $U_{d}$ at the wall is given by:

$$
U_{d}=-\left.\frac{\nu}{\omega} \frac{\partial \omega}{\partial y}\right|_{w}
$$

then the vorticity gradient at the wall is given by:

$$
\left.\frac{\partial \omega}{\partial y}\right|_{w}=-\frac{1}{\nu} \frac{d U_{s}}{d t}=-\left.\frac{1}{\nu} \frac{d \gamma}{d t}\right|_{w}
$$

We have examined several numerical models for introducing (diffusing) the vorticity into the flow at the wall. In a recent paper by Koumoutsakos, Leonard and Pepin [8] concerning vorticity generation at a wall, an algorithm for updating the particle strengths in a PSE method was presented for finite panels with curvature. In the context of the present unidimensional model, the vorticity which would be introduced into the domain from the wall during a time $\Delta t$ would have a distribution equal to:

$$
\Delta \omega=\omega^{t+\Delta t}-\omega^{t}=\frac{U_{s} e^{-\frac{y^{2}}{4 \nu \Delta t}}}{\sqrt{\pi \nu \Delta t}}
$$

where $\omega^{t+\Delta t}$ is the vorticity at the time $t+\Delta t$ and $\omega^{t}$ is the vorticity at time $t$. We note that Eq. 19 is identical to the exact solution for an impulsively started flat plate [4]. Adding all of the circulation to the first element at the beginning of the time step is roughly equivalent to using Eq. 19 at the end of the time step.

Since the diffusion equation is linear, we may obtain the vorticity distribution associated with the flux from the wall at the end of the time step by superimposing the impulsive solutions via the following convolution integral.

$$
\Delta \omega=\int_{0}^{\Delta t} \frac{\partial U_{s}}{\partial \tau} \frac{\exp \left(-\frac{y^{2}}{4 \nu(\Delta t-\tau)}\right)}{\sqrt{\pi \nu(\Delta t-\tau)}} d \tau
$$


Assuming that the slip velocity changes linearly as a function of time over the time step $\Delta t$ and defining $y^{+} \equiv y / \sqrt{\nu t}$, Eq. 20 can be integrated numerically to obtain the curve shown in Fig. 3 which is labeled as "Exact". The resulting distribution can be fitted with a curve given by:

$$
\Delta \omega^{+} \equiv \frac{\Delta \omega \sqrt{\frac{\nu}{\Delta t}}}{\frac{\partial U_{s}}{\partial \tau}}=1.131 e^{-q}
$$

where

$$
q=0.884 y^{+}+0.161 y^{+2}
$$

For comparison, the distribution given by a single impulse at the mid time step is also plotted in Fig. 3 identified in the legend by $0.5 \Delta t$. The major difference in the two curves is their distributions near the wall. From Eq. 18 it is seen that the slope of the $\left(\Delta \omega^{+}, y^{+}\right)$curve at the wall must be equal to minus one, not zero as is given by the impulsive start representation. We note that the proper slope is obtained when using Eq. 21. For simulations where $\Delta t \Rightarrow 0$, the global vorticity distribution will tend to be the same for the various representations of the wall flux distribution. On the other hand, we have found that one has to sometimes take much smaller time steps when using the impulsive representation in order to obtain satisfactory distributions near the wall. We therefore recommend the use of Eq. 21.

\subsection{The diffusion velocity for zero vorticity}

In order to use Eq. 12, it is important to obtain an understanding of its behavior for the case where $\omega=0$. We note that there are two basic zero vorticity cases which must be considered. In the first case, the vorticity and its slope are both approaching zero. In the second case, the vorticity is zero but the slope is non-zero. We first examine the case where the vorticity and its slope are both approaching zero. The diffusion velocity in the region where $\omega \rightarrow 0$ is indeterminate for this case since $\partial \omega / \partial y$ is also approaching zero. In order to form a numerical model for this case, we assume a linear vorticity distribution as shown in Fig. 4. Since our primary interest is in the diffusion of this linear element, we write an equation for the mean diffusion velocity $U_{d M}$ at $y=h / 2$ and equate that to the average of the diffusion velocities at the ends of the element.

$$
U_{d M}=\frac{U_{d L}+U_{d 0}}{2}=-\frac{2 \nu}{\omega_{L}}\left(-\frac{\omega_{L}}{h}\right)
$$

Solving Eq. 23 for $U_{d 0}$ yields:

$$
U_{d 0}=4 \frac{\nu}{h}-U_{d L}
$$

To test the applicability of Eq. 24, we have examined its predictive quality with regard to diffusion of vorticity in a flow in which the wall is impulsively started from rest to a constant velocity. The entire vorticity field is modeled with a single linear element, which is of course a very crude model for such a flow. In an actual simulation, this linear element would represent only a small region of the flow at the edge of the diffusing layer. The vorticity distribution obtained from the one element linear approximation is surprisingly close to that of the exact solution and in fact, fluid velocity profiles obtained by integrating these curves would appear to be even more similar. Based on this limited test, we conclude that Eq. 24 should give satisfactory results for representing an element (in a general numerical model) where $\omega$ and $\partial \omega / \partial y$ are both approaching zero.

Next, consider the case depicted in Fig. 5 in which $\omega=0$ but $\partial \omega / \partial y \neq 0$. From Eq. 12, it may be seen that there is a jump in the value of $U_{d}$ from a very large positive value to a very large 
negative value as one moves from left to right across the $\omega$ axis along the $y$ axis. This indicates that negative vorticity is flowing to the right while positive vorticity flows to the left. The total strength of the vortex sheet remains constant with respect to time but the vorticity sheet strength $\gamma_{R}$ for the right hand layer is decreasing, on the other hand, at a rate given by:

$$
\frac{d \gamma_{R}}{d t}=-\nu \frac{\partial \omega}{\partial y}
$$

while the vorticity sheet strength $\gamma_{L}$ for the left hand layer is increasing (in a positive sense) at an equal rate. Thus, we observe that positive and negative vorticity is being destroyed in equal amounts.

We have examined several numerical models for dealing with this problem. The easiest method is to simply break the vorticity distribution up into positive and negative distributions, diffuse them independently, and then add them back together. This is permissible since the diffusion process is linear. The cancellation of positive and negative vorticity occurs during the addition process and does not otherwise have to be accounted for. We found this method to be very robust and have used it in all of our calculations.

\section{Numerical results}

\subsection{Initially uniform vorticity sheet}

For this case, an infinite plate moves at a constant velocity of $U_{e}$ to the left. Initially, at time $t=0$, the tangential velocity at the plate is equal to $U_{e}$ and zero at a distance $h$ away from the plate. The profile is linear. We do not concern ourselves with how this profile might have been generated but simply specify it as an initial condition. The linear velocity between $y=0$ and $y=h$ at $t=0$, implies that there is a layer of vorticity of thickness $h$ next to the surface. The vorticity in this layer is constant and has a value of $\omega=U_{e} / h$ in the counter clockwise direction. The exact analytical solution for this case is given by:

$$
\omega^{*}=\frac{1}{2}\left[\operatorname{erf}\left(\frac{1-y^{*}}{2 \sqrt{t^{*}}}\right)+\operatorname{erf}\left(\frac{1+y^{*}}{2 \sqrt{t^{*}}}\right)\right]
$$

where, $\omega^{*}=\omega h / U_{e}, y^{*}=y / h$, and $t^{*}=\nu t / h^{2}$.

Plots of the vorticity distribution as a function of time using the computer code BDIF are given in Fig. 6 as the open symbols. The "exact" solution (Eq. 26) is also plotted in Fig. 6 as a solid line. We have removed approximately $50 \%$ of the points calculated by BDIF to enable one to be able to compare with the exact solution. The results using the diffusion velocity are seen to be in excellent agreement with the analytical results. The biggest error occurs at the edge of the boundary layer where $\omega$ is small and the diffusion velocity is large.

\subsection{Impulsively started plate}

We next examine the impulsively started plate sometimes referred to as "Stokes' First Problem". The exact solution for the velocity distribution can be obtained from Currie [4]:

$$
\frac{u}{U_{e}}=1-\operatorname{erf}\left(\frac{y}{2 \sqrt{\nu t}}\right)
$$


By differentiating Eq. 27 with respect to $y$, the vorticity field is readily obtained:

$$
\frac{\omega \sqrt{\nu t}}{U_{e}}=\frac{1}{\sqrt{\pi}} \exp \left(-\frac{y^{2}}{4 \nu t}\right)
$$

In Fig. 7 we show a calculation using the code BDIF in which the plate was accelerated from a zero velocity to the velocity $U_{e}$ in a time step that is equal to $0.001 t$. The exact calculation (Eq. 28) is plotted for comparison. Again, we have removed approximately $50 \%$ of the points calculated by BDIF. These results show excellent agreement between the exact solution and the BDIF calculation.

\subsection{Sinusoidally moving plate}

We next examine the sinusoidally oscillating plate which is sometimes referred to as "Stokes Second Problem". Wu et al. [15] and Panton [11] address the issue of transient vorticity values for this problem. By "transient" we mean the start-up period prior to a periodic solution. One method of solving both the transient and periodic problem, is to use the convolution integral given by Eq. 20 with the slip velocity prescribed by the plate motion. This method is valid in the present case since there is no net accumulation of vorticity due to convection at any stream-wise location. We have developed a simple computer code to numerically integrate this equation and refer to this method as the convolution integral method or CIM. In Fig. 8, the vorticity profiles in the eleventh cycle of oscillation are shown. In this figure the CIM simulations form our baseline calculations and are denoted by the solid lines. The BDIF code was used to produce the data denoted by the symbols. The agreement is seen to be very good.

\subsection{Flat plate flow (Blasius solution)}

The next solution which we will examine is the case for flow over a finite length flat plate. This solution was generated by the code WALLYR which represents an exploratory implementation of the solution for wall layer flows as outlined earlier. In this particular calculation, the size of the wall computational space and Reynolds number were chosen to contain all of the vorticity within the wall region $(y \leq h)$ and, hence, there was no need for any of the vorticity to be represented by free two-dimensional blobs. Rather, we treat the entire boundary layer as a wall flow in which vorticity is introduced at the wall, diffused, and convected. The plate was discretized into 50 elements with $h=1$.

The solution was started with the plate moving to the left with a velocity of $U_{o}=1$. The initial vorticity distribution was assumed to be uniform along the length of the plate and was computed using Eq. 19 with $\Delta t=1$. Numerical experimentation revealed, however, that the starting solution did not affect the final results. For the results shown here, the solution converged at a non-dimensional time $t=200$.

The results of the calculations for the vorticity profile at a Reynolds number based on the length from the plate's leading edge, $R e_{x}$, of $1 \times 10^{5}$ are shown in Fig. 9 along with the exact solution given in Schlichting [14]. Solutions at Reynolds numbers of $0.8 \times 10^{5}, 1.6 \times 10^{5}, 2 \times 10^{5}, 2.4 \times 10^{5}$, and $3.2 \times 10^{5}$ were also calculated. Calculated stream-wise and transverse velocity profiles also showed excellent agreement with the exact solutions.

\subsection{Wedge flows}

We have computed solutions for several wedge flows with different wedge angles using the computer code WALLYR. The "exact solution" was obtained from the similarity equations associated with 
wedge flows which are themselves special cases of the Falkner-Scan similarity equations found in many fluid mechanics texts such as [4] and [14]. The velocity which is tangent to the wall just outside of the boundary layer is given by $u=c x^{m}$. The wedge half angle $\theta$ is related to the value of $m$ by:

$$
\theta=\pi\left(\frac{m}{m+1}\right)
$$

In Fig. 10, we present the vorticity profile for a wedge flow with $\theta=30^{\circ}$. The agreement is seen to be excellent. Calculations for stream-wise and transverse velocity profiles also yielded good results with a small deviation from the exact solution for the transverse velocity profile near the edge of the boundary layer. Calculations made at several stream-wise locations yielded similar results.

\subsection{Two-dimensional diffusion}

The last case which we have calculated is a pure two-dimensional diffusion problem for an initially square patch of uniform vorticity. The initial non-dimensional lengths of the patch are set equal to 1.0 as is the value of the vorticity on the patch. The patch is discretized by 2500 Gaussian vortex blobs which have a core radius of $1 / 50$. Each of the blobs are overlapped by one core radius. The numerical simulation was conducted out to a non-dimensional time $t^{+}=\nu t / l^{2}=4$, where $l$ is the initial length of the sides of the patch. As can be seen from Fig. 11, the maximum value of vorticity in the distribution at $t^{+}=4$ is only $8 \%$ of that at $t^{+}=0$.

An exact solution for this problem is found in Carslaw and Jaeger [1]. In order to compare the two methods, a 200 by 200 evaluation grid was first constructed on a 20 by 20 patch containing the original 1 by 1 patch at its center. Comparison of the two methods was then made by forming an RMS error value based on the error at the 40000 evaluation points. The RMS error normalized by the peak vorticity value at any instant in time was found to be approximately $0.01 \%$.

\section{Summary}

- We have developed a set of algorithms for treating laminar diffusion near a wall using the diffusion velocity concept. These algorithms have the following concepts embodied in them:

- An accurate algorithm for representing the flux and distribution of vorticity generated at the wall during a given time step has been developed.

- Due to the linear nature of the diffusion equations we can treat the diffusion of oppositely signed vorticity separately and thus do not have to deal with the conceptually difficult situation where the vorticity is zero while the gradient of vorticity is not.

- "Diffusion boundaries" across which existing vorticity cannot flow are easily produced by using images of the vorticity distribution.

- We have demonstrated that very good results are obtainable for the diffusion of a finite thickness vortex sheet, an impulsively started flat plate, an oscillating flat plate, uniform flow over a semi-infinite flat plate, and flow over a wedge.

- We have developed a set of algorithms for treating two-dimensional diffusion in free space using the diffusion velocity concept. Very accurate results have been obtained from a simulation in which an initially square patch of vorticity was allowed diffuse. 
- There is a need for further development and demonstration of the "bi-zonal concept" such that a smooth coupling and transition between the inner and outer layers can be achieved.

- In order to deal with high Reynolds number flows near walls, the effect of small scale turbulence must be included. This should include an attempt to apply existing turbulence models as well as investigate concepts which might be better suited to a vorticity based method.

\section{References}

[1] Carslaw, H.S. and Jaeger, J.C., Conduction of Heat in Solids, Oxford University Press, pp. 176-187, 1984.

[2] Chorin, A.J., "Numerical Study of Slightly Viscous Flow," J. Fluid Mech., 57, pp. 785-796, 1973.

[3] Chorin, A.J., "Vortex Sheet Approximation of Boundary Layers," J. Comp. Phys., 27, pp. 428-442, 1978.

[4] Currie, I.G., Fundamental Mechanics of Fluids, McGraw-Hill, pp. 231-237, 1974.

[5] Fishelov, D., "A New Vortex Scheme for Viscous Flows," J. Comp. Phys., 86, pp. 211-224, 1990.

[6] Kempka, S.N. and Strickland, J.H., "A Method to Simulate Viscous Diffusion of Vorticity by Convective Transport of Vortices at a Non Solenoidal Velocity," Sandia Laboratory Report SAND93-1763, 1993.

[7] Kempka, S.N. and Strickland, J.H., "Velocity and Outflow Boundary Conditions for Vorticity Formulations," Sandia Laboratory Report SAND94-1735, 1995.

[8] Koumoutsakos, P., Leonard, A. and Pepin, F., "Boundary Conditions for Viscous Vortex Methods," J. Comp. Phys., 113, pp. 52-61, 1994.

[9] Leonard, A., "Vortex Methods for Flow Simulation," J. Comp. Phys., 37, pp. 289-335, 1980.

[10] Ogami, Y. and T. Akamatsu, "Viscous Flow Simulation using the Discrete Vortex Model—The Diffusion Velocity Method," Comp. Fluids, 19, pp. 433-441, 1991.

[11] Panton, R., "The Transient for Stokes's Oscillating Plate: A Solution in Terms of Tabulated Functions," J. Fluid Mech., 31, pp. 819-825, 1968.

[12] Spalart, P.R., "Vortex Methods for Separated Flows," NASA TM 100068, 1988.

[13] Spalart, P.R., Leonard, A., and Baganoff, D., "Numerical Simulation of Separated Flows," NASA TM 84328, 1983.

[14] Schlichting, H., Boundary Layer Theory, McGraw-Hill, pp. 125-129, 1968.

[15] Wu, J.Z., Wu, X.H., Ma, H.Y. and Wu, J.M., "Dynamic Vorticity Condition: Theoretical Analysis and Numerical Implementation," Intl. J. for Num. Meth. in Fluids, 19, pp. 905-938, 1994. 


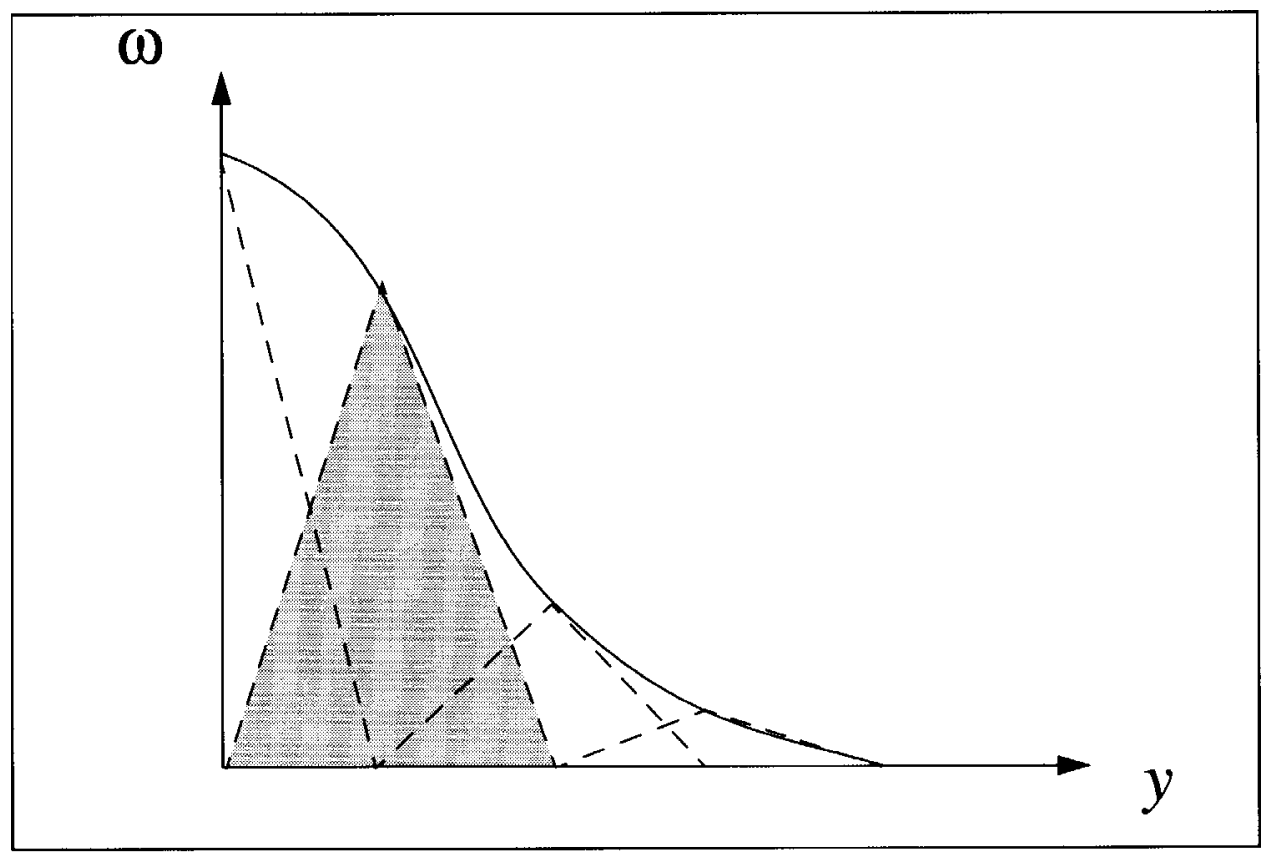

Figure 1: Diffusion elements near wall.

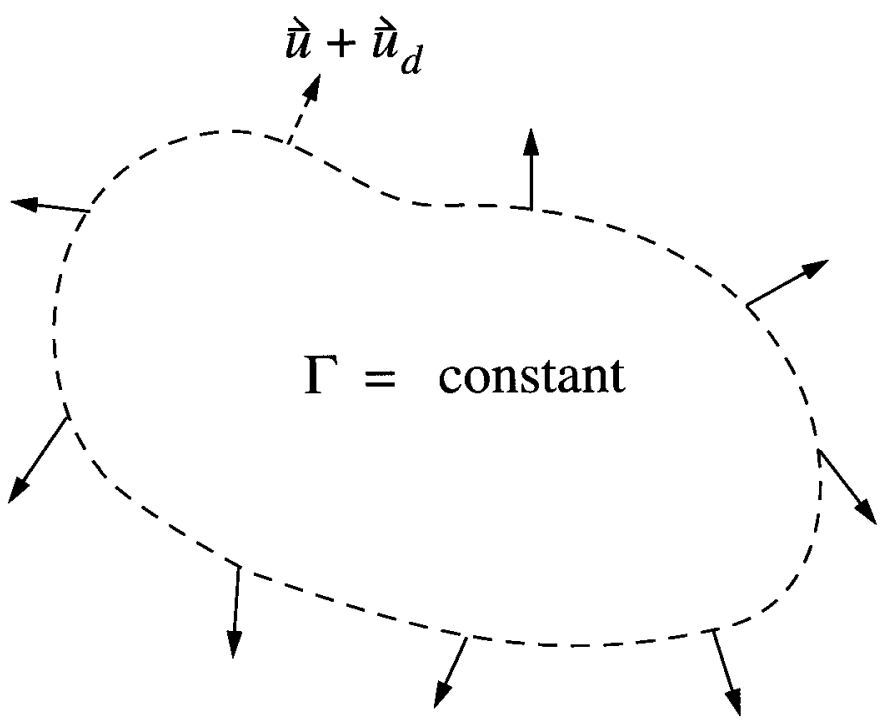

Figure 2: Diffusion velocity concept. 


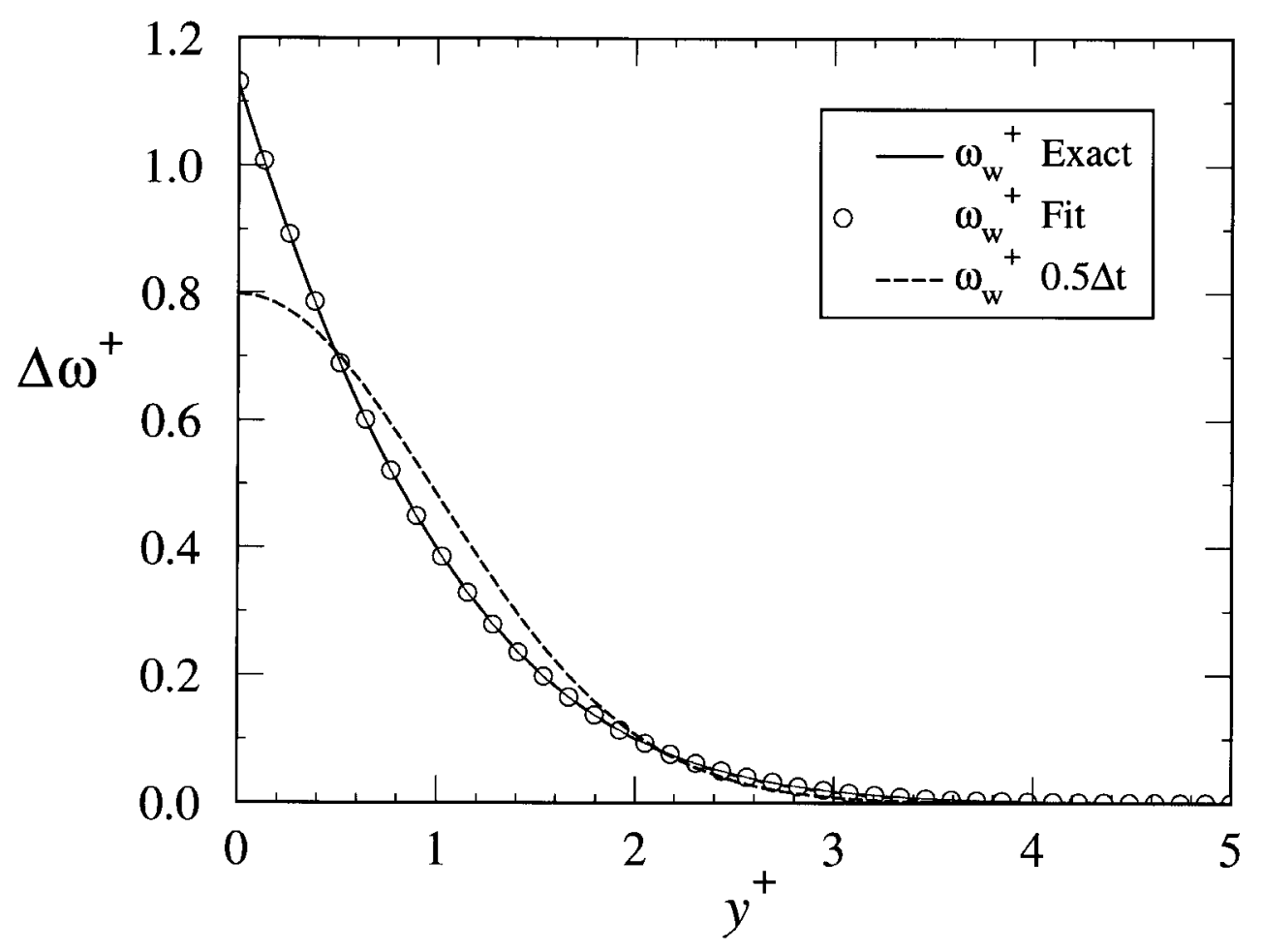

Figure 3: Distribution of wall vorticity.

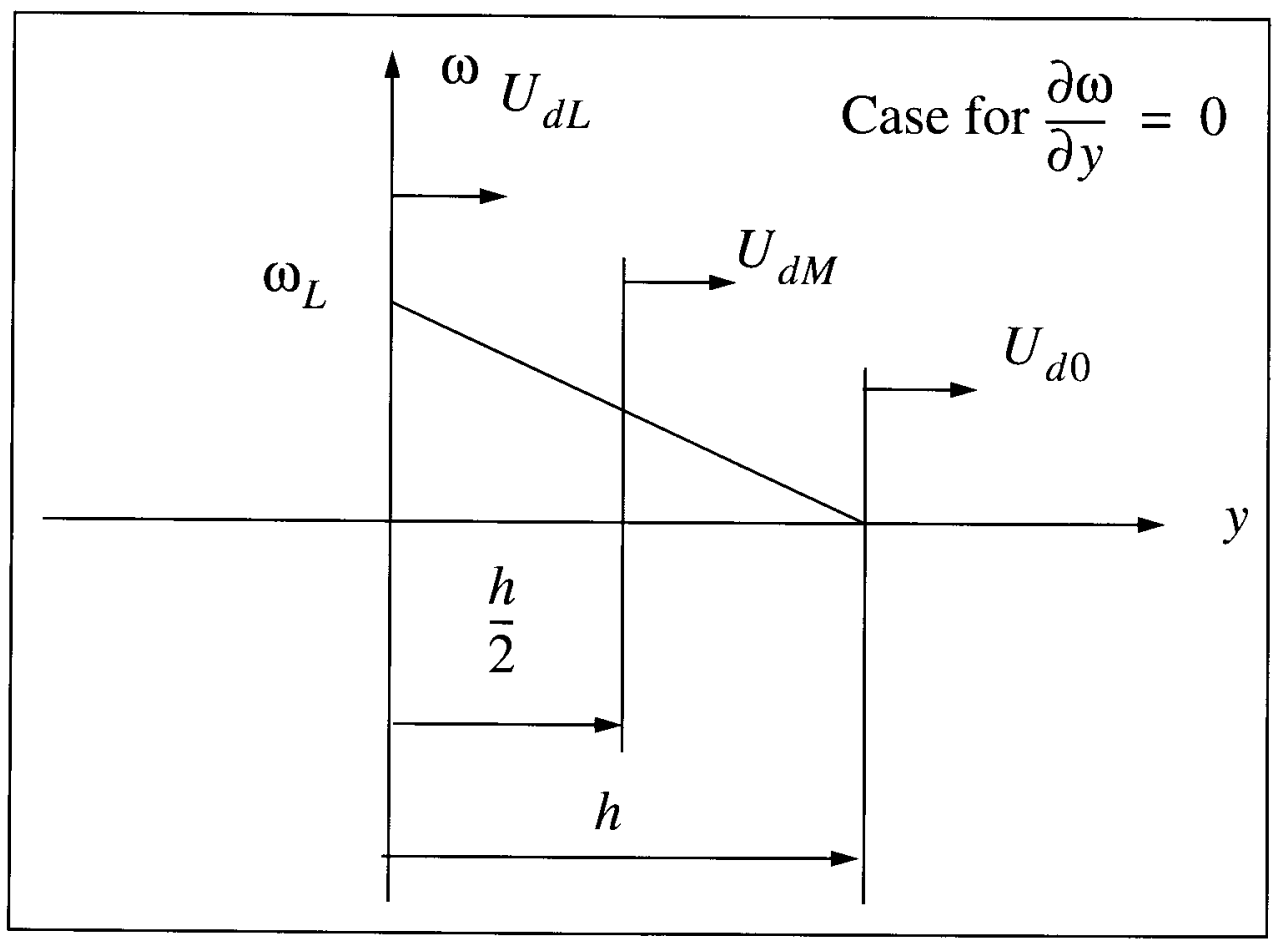

Figure 4: Zero vorticity case. 
()

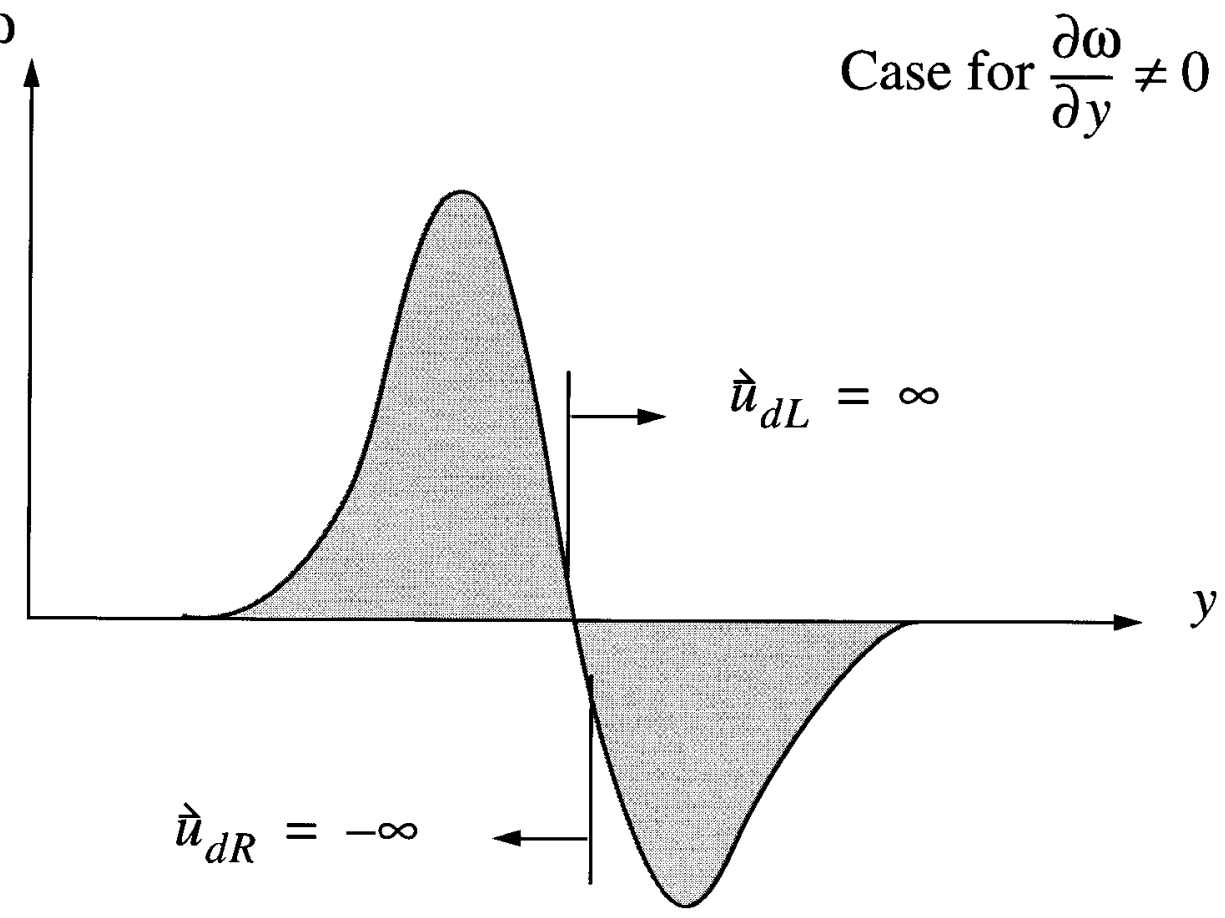

Figure 5: Zero vorticity case.

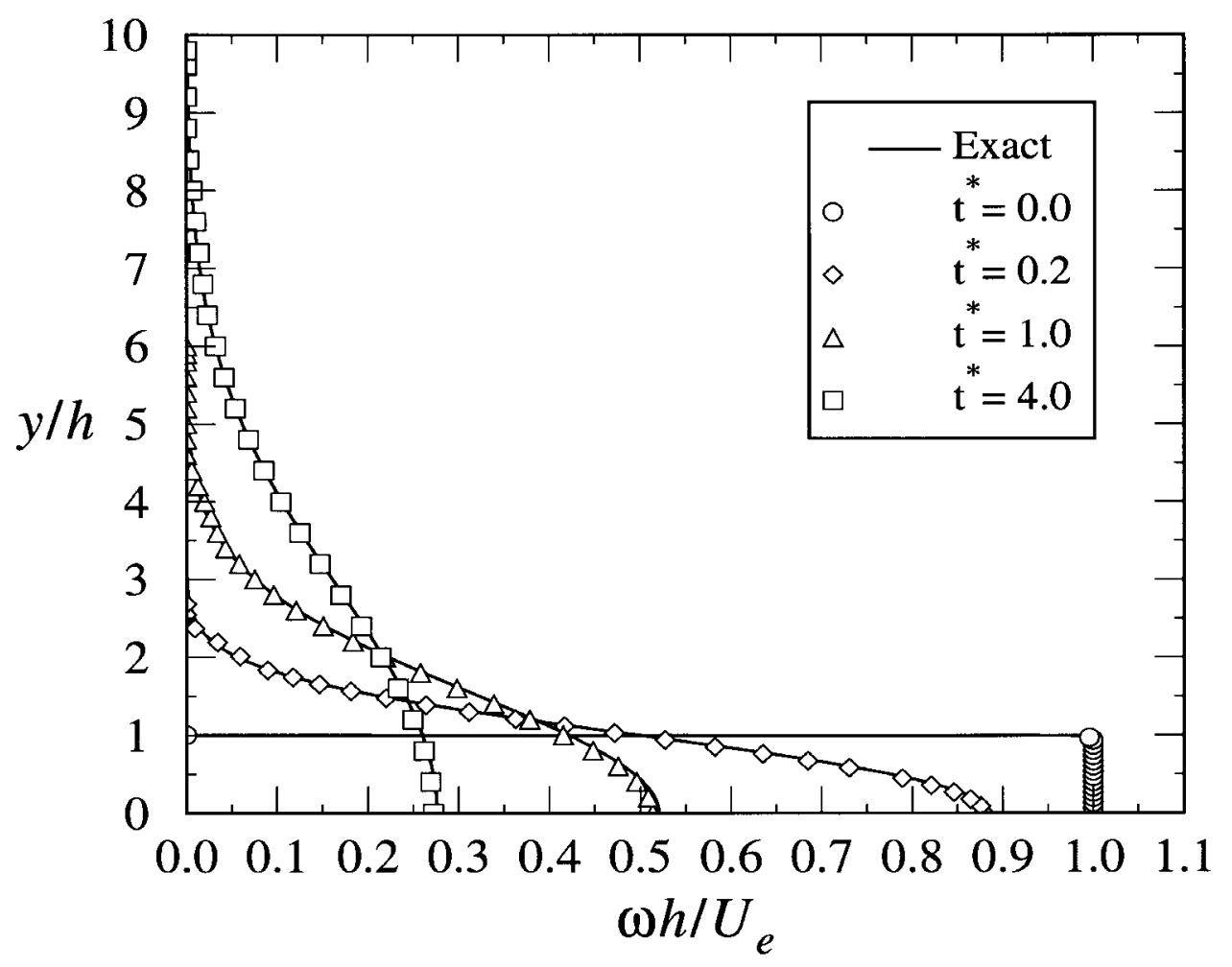

Figure 6: Initially uniform vorticity sheet. 


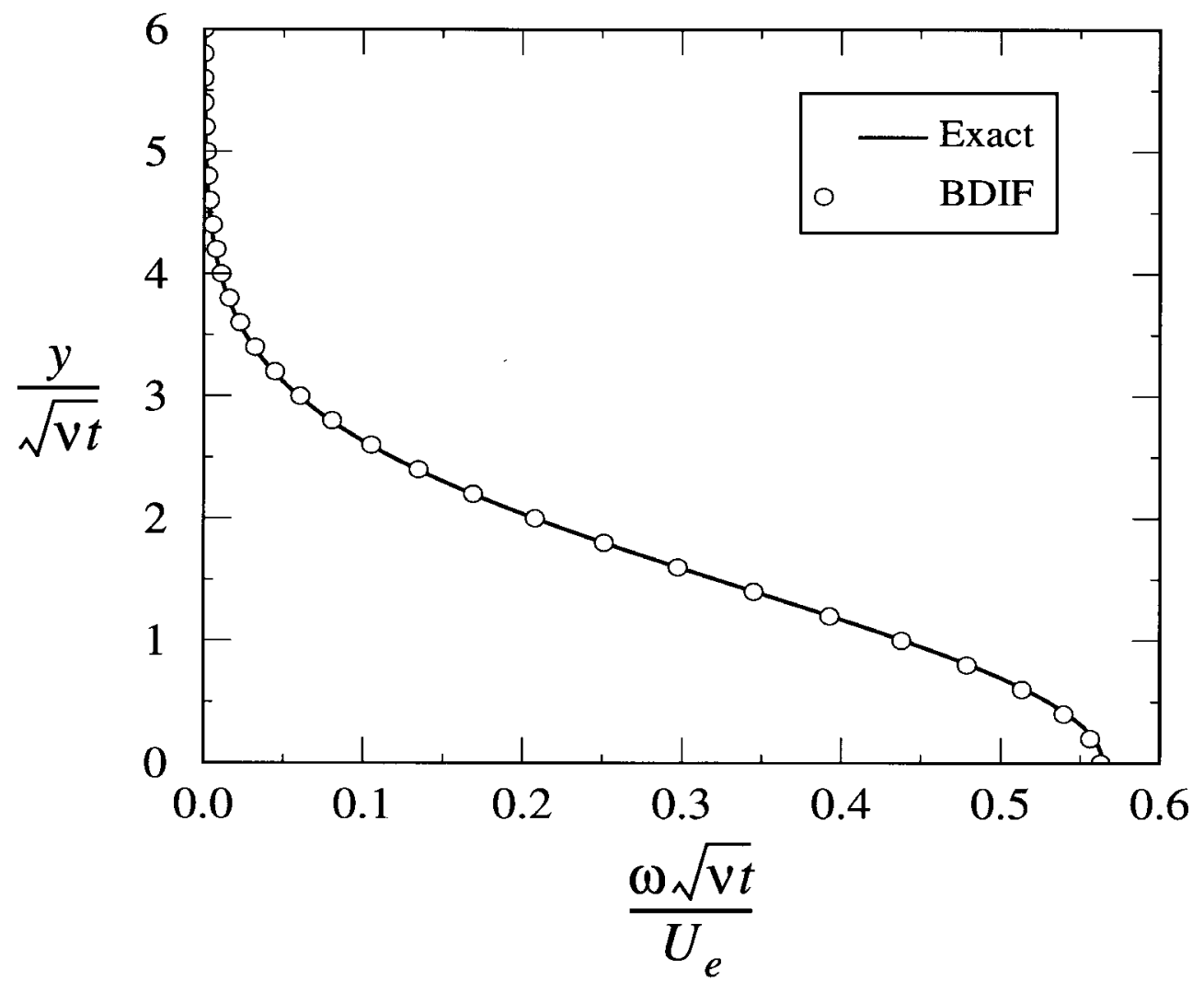

Figure 7: Impulsively started plate. 


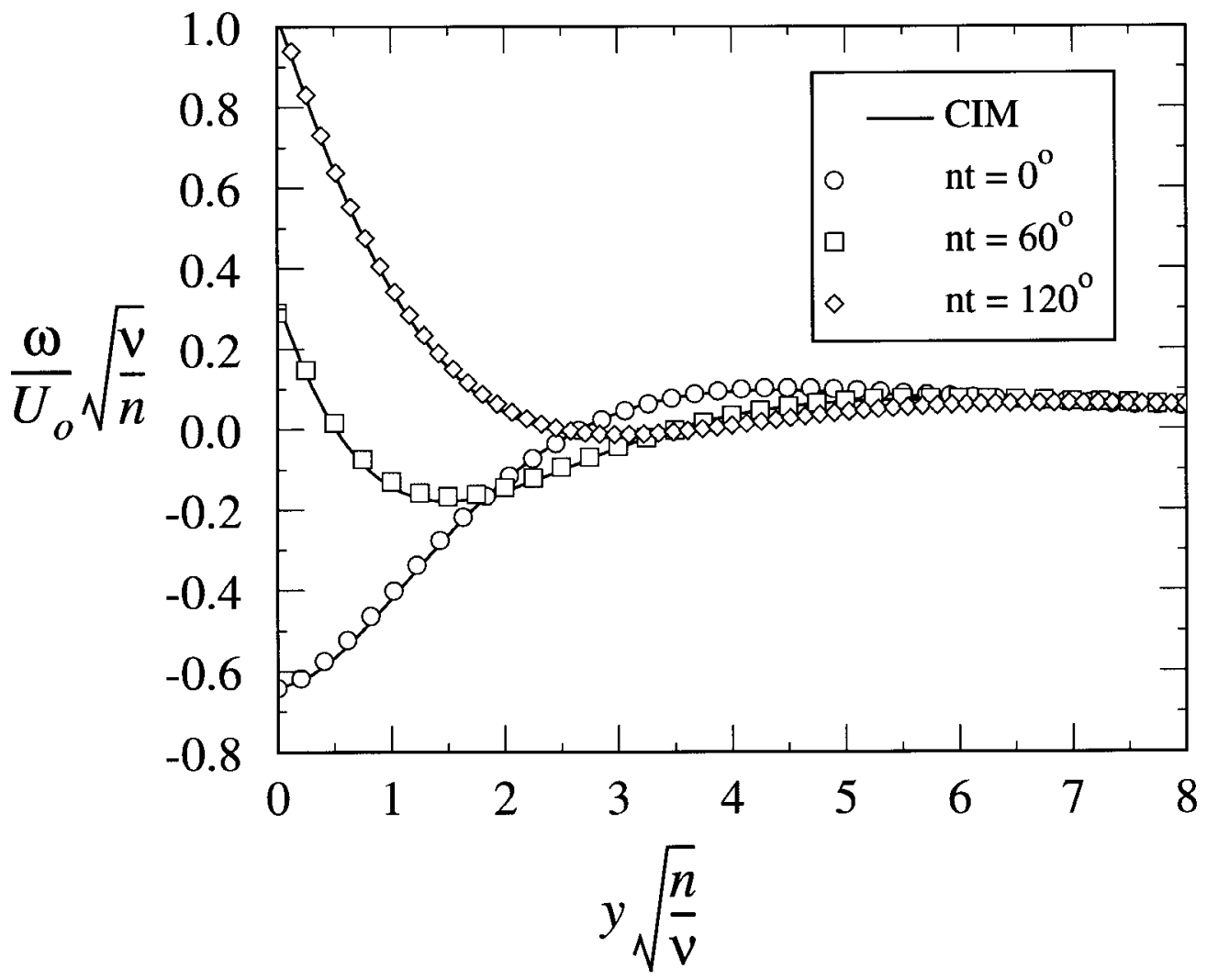

Figure 8: Sinusoidally oscillating plate. 


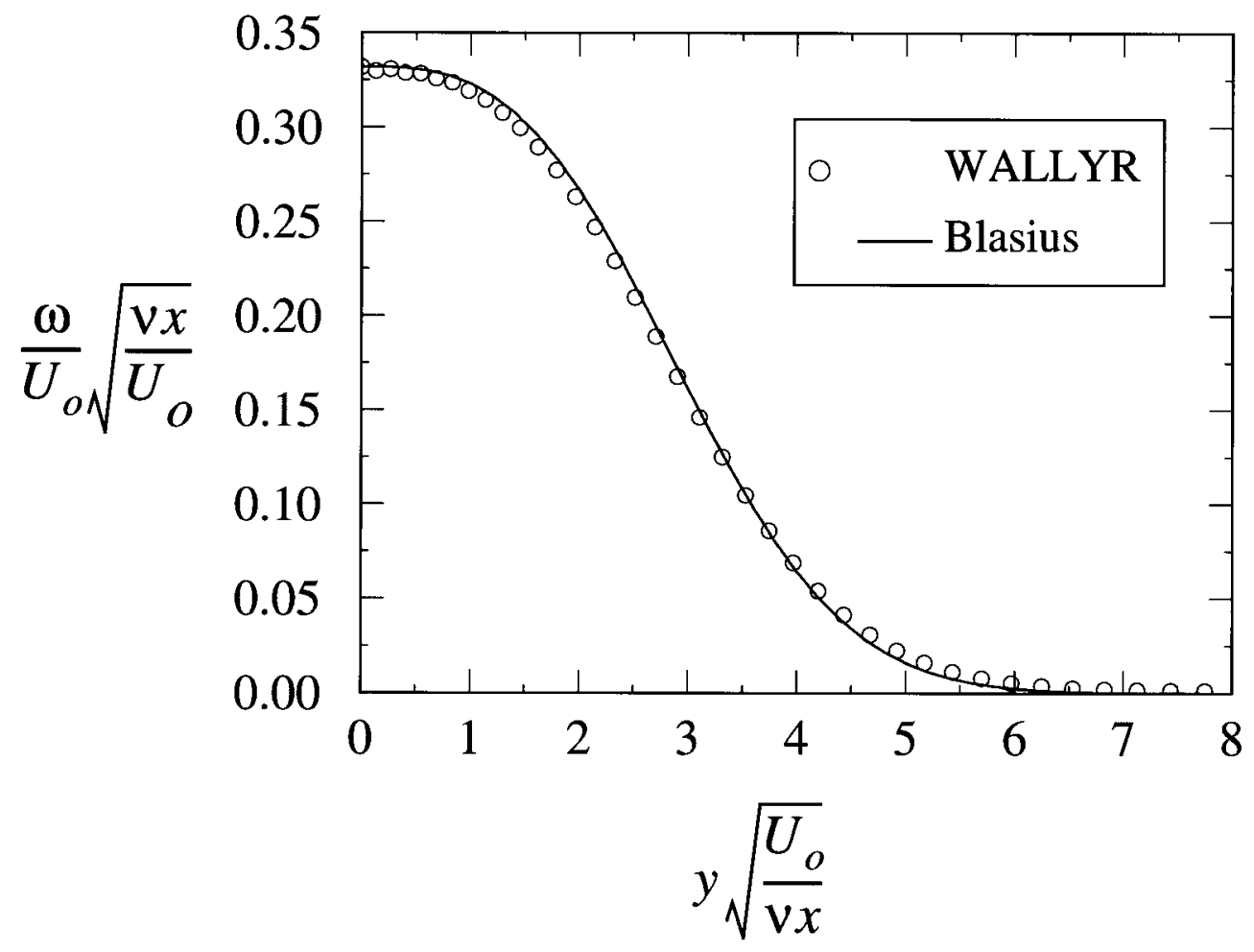

Figure 9: Flat plate (Blasius).

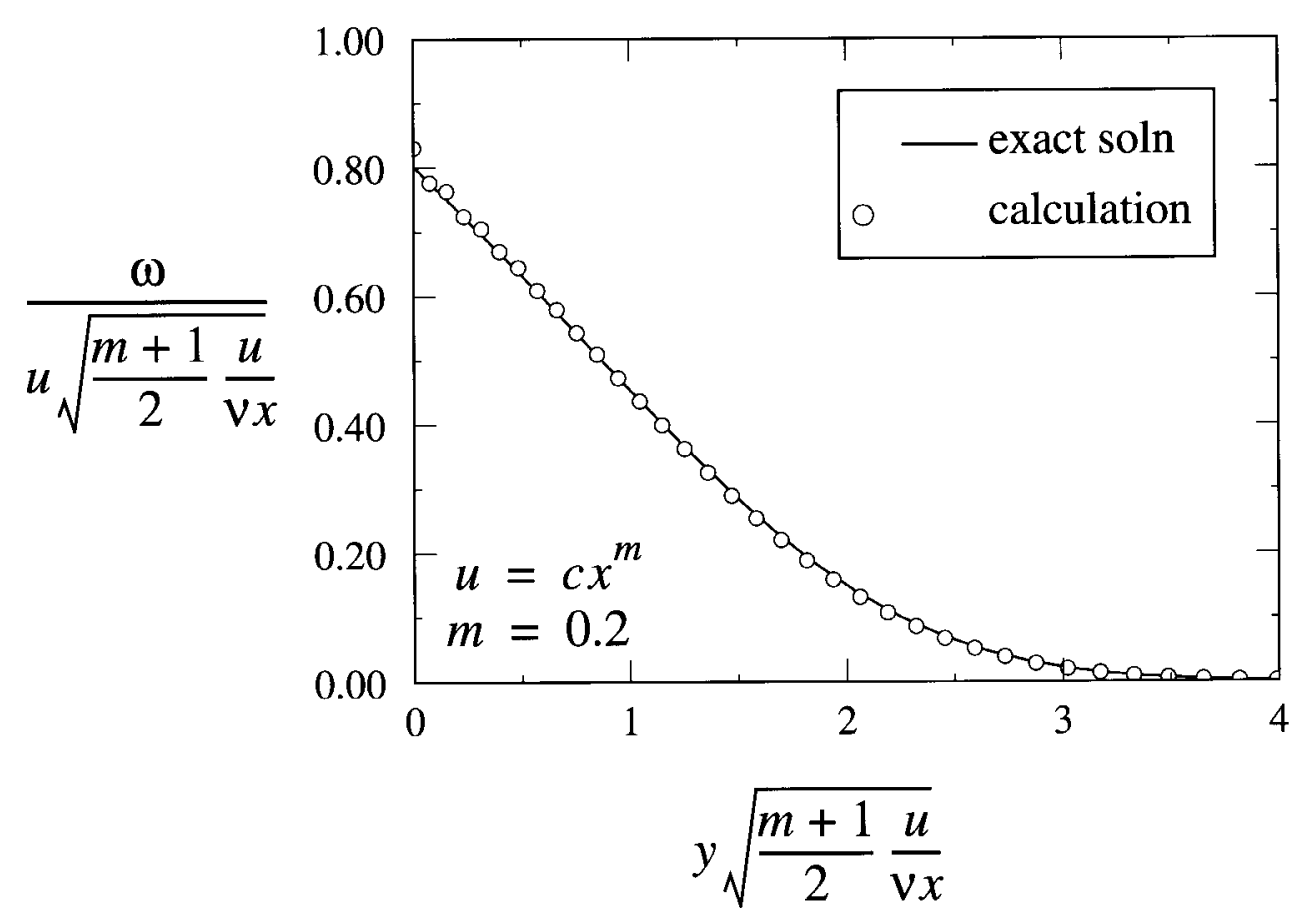

Figure 10: Wedge flow. 


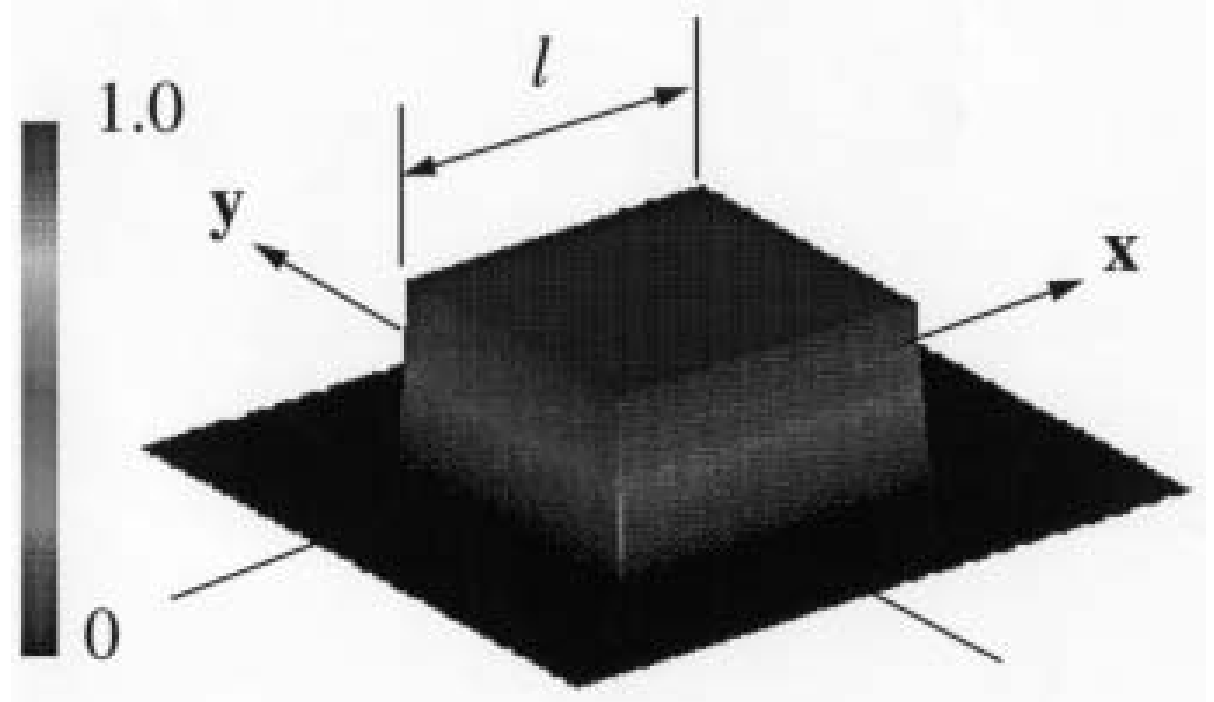

$$
\frac{v t}{l^{2}}=0
$$

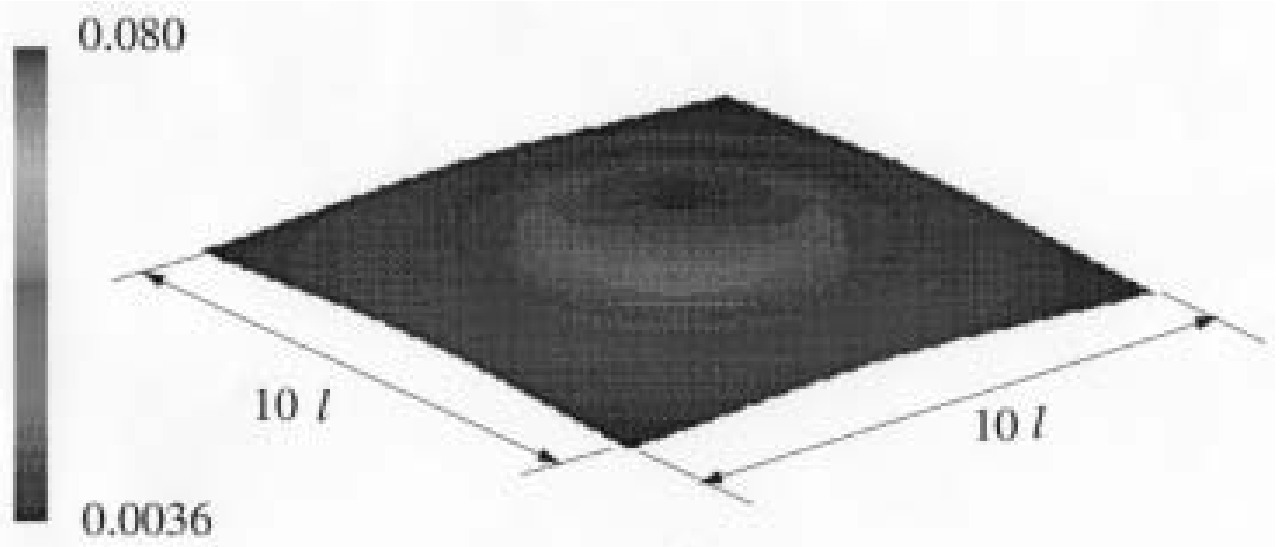

$$
\frac{v t}{l^{2}}=4
$$

Figure 11: Two-dimensional lagrangian diffusion 